\title{
A New Algorithm for Black-start Zone Partitioning Based on Fuzzy Clustering Analysis
}

\author{
Yujia Li ${ }^{1}$, Yu Zou ${ }^{1}$, Yupei Jia ${ }^{1}$, Yunxia Zheng ${ }^{2}$ \\ ${ }^{1}$ China Electric Power Research Institute, Beijing, China \\ ${ }^{2}$ Jibei Qinhuangdao Electric Power Company, Hebei, China \\ Email: liyujia@epri.sgcc.com.cn, zhengyunxia509@sina.com
}

Received April, 2013

\begin{abstract}
On the process of power system black start after an accident, it can help to optimize the resources allocation and accelerate the recovery process that decomposing the power system into several independent partitions for parallel recovery. On the basis of adequate consideration of fuzziness of black-start zone partitioning, a new algorithm based on fuzzy clustering analysis is presented. Characteristic indexes are extracted fully and accurately. The raw data matrix is made up of the electrical distance between every nodes and blackstart resources. Closure transfer method is utilized to get the dynamic clustering. The availability and feasibility of the proposed algorithm are verified on the New-England 39 bus system at last.
\end{abstract}

Keywords: Black-start Zone Partitioning; Fuzzy Clustering Analysis; Electrical Distance; Closure Transfer Method

\section{Introduction}

Since the 1960s many power outages have occurred arround the world, which brought great losses to both social production and people's livelihood [1-4]. After the blackout, the black-start power needs to provide power for the units without self-starting capability as little time as possible so that they can reconnect the grid and supply the main node, and gradually establish a preliminary recovery which lay the foundation of fully load recovery in next stage. During the recovery process, taking partitioning recovery, by which each subsystem recovers independently, is not only conducive to shorten recovery time-consuming but also help to improve the security of the entire system recovery.

It is beneficial to ensure the smooth progress of partitioning recovery that adopting a scientific partitioning algorithm. Reference [5] has discussed system partitioning based on the distribution of black-start generators considering the startup characteristics of the unit and system status, but lack of consideration of restrictions for the security and the matching between power generation and load. In [6] a new subsystem partitioning algorithm based on theory of structure of complex network has been proposed. There is a certain degree of subjectivity for the target unit which starts after black-start unit has been determined and nodes of the path between the black-start unit and target unit have been combined into one node before calculation. Reference [7] proposed a black-start zone partitioning strategy based on ordered binary decision diagram trying to obtain optimal solution mainly by topology discovery, but other influencing factors were concerned too less.

In this paper, a black-start zone partitioning algorithm based on fuzzy clustering analysis theory is proposed with the aim to avoid deficiencies for the current methods of black-start zone partitioning. The reliability and timeliness of the black-start recovery has been conesdered fully on the extracting of the features indicators. The electrical distance between the target unit, load bus bars, important stations and black-start units have been defined to form the original data matrix, and the transitive closure method for clustering has been adopted. The verification results show that the method is feasible and effective.

\section{Fuzzy Clustering Analysis}

Cluster analysis is one of multivariate statistical analysis. It groups a set of objects in such a way that objects in the same group (called cluster) are more similar (in some sense or another) to each other than to those in other groups (clusters)

Traditional cluster analysis is a kind of "hard clustering”. Each object is identified strictly and is divided in certain types of "either-or" nature. In fact most of the object does not have strict property and "soft clustering" is more suitable [8]. Fuzzy Clustering Analysis is a 
method of "soft clustering" which considering not only whether the relationship between objects exists but also the degree of the relationship by taking full account of the fuzziness of objects being analysed. Fuzzy clustering analysis has been widely used in many fields of power system such as load characteristics analysis [9], failure diagnosis [10], electricity prices partition [11], voltage control partition [12], except the filed of black-start zone partitioning.

\subsection{Steps of Fuzzy Clustering Analysis}

Steps of fuzzy clustering analysis can be summarized as follows:

a) original data matrix building;

b) normalization;

c) demarcating;

d) clustering.

1) original data matrix building

Assuming that set are objects need to classified, each object consists of $n$ indicators that can describe its traits and the n-th indicator is denoted as so that the original data matrix can be established as. It should be noted that indicators in the matrix need to be determined with specific analysis for the certain problem and that extracting characteristic indicators fully and accurately is the basis for the application of fuzzy clustering method.

2) Normalization

When solving practical problems different data generally have different dimensions. Some characteristic indicators with particularly large magnitude may contribute to classifying too much while those with the very small magnitude may be ignored during the calculating. In order to make data with different dimension can be compared with each other; we usually need to transform data in an appropriate way. In accordance with the requirements of the fuzzy matrix, data should be compressed into the interval $[0,1]$. Method of data normalization applied more frequently at present are as follows: zeromean normalization, min-max normalization, max normalization, mean normalization, center normalization, logarithmic normalization, etc. This paper takes the minmax normalization method to normalize the original data matrix, by which the expression is shown below,

$$
y_{i k}=\frac{x_{i k}-\min _{1 \leq i \leq m}\left\{x_{i k}\right\}}{\max _{1 \leq i \leq m}\left\{x_{i k}\right\}-\min _{1 \leq i \leq m}\left\{x_{i k}\right\}}, \quad(k=1,2, \cdots, n)
$$

Obviously, $0 \leq y_{i k} \leq 1$, and the influence of dimensions of each indicator has been removed after normalization.

3) Demarcating

Demarcating is aim to get the statistics which can measure the degree of similarity of objects being classi- fied. Assuming that Interval discussed is given below:

$$
\begin{aligned}
& X=\left\{x_{1}, x_{2}, \cdots, x_{m}\right\}, x_{i}=\left(x_{i 1}, x_{i 2}, \cdots, x_{i n}\right) \\
& (i=1,2, \cdots, m)
\end{aligned}
$$

Meanwhile, the degree of similarity between $x_{i}$ and $x_{j}$ is defined as $r_{i j}=R\left(x_{i}, x_{j}\right),(i, j=1,2, \cdots, m)$. Methods to get $r_{i j}$ mainly include similarity coefficient method (such as scalar product method, cosine method, exponential similarity coefficient method, correlation coefficient method, max and min method, min arithmetic average method, and min geometric mean method), distance method (such as absolute value reciprocal method, absolute value exponential method, and direct distance method), subjective evaluation methods, etc. We take absolute value exponential method to calculate $r_{i j}$ which is given by:

$$
\begin{aligned}
& r_{i j}=\exp \left(-c \sum_{k=1}^{n}\left|y_{i k}-y_{j k}\right|\right), \\
& (i, j=1,2, \cdots, m)
\end{aligned}
$$

where $c$ is a specific positive number range from 0 to $1\left(0 \leq r_{i j} \leq 1\right)$, in this case we set $c=1$.

4) Clustering

Clustering is the process to classify the object of study based on the fuzzy matrix. Clustering method mainly include clustering based on equivalent fuzzy matrix, direct cluster and maximal tree. Closure transfer method is adopted in this paper and the detail will be shown in next part.

\subsection{Fuzzy Clustering Transitive Closure}

The calibration matrix $R$ obtained before is just a fuzzy similar matrix and not necessarily transitive, therefore, we should transform $R$ into fuzzy equivalent matrix $R^{*}$. Quadratic formulation is used here to calculate transitive closure of $R$ so that equation $t(R)=R^{*}$ can be expressed as:

$$
R \rightarrow R^{2} \rightarrow R^{4} \rightarrow \cdots \rightarrow R^{2 k} \rightarrow \cdots
$$

After a finite number of computing, there is a $\mathrm{k}$ which fit the equation $R^{2 k}=R^{k} \circ R^{k}=R^{k}$ indicate that $R^{k}$ has been transitive already, meanwhile, $R^{*}=R^{k}$ is the fuzzy equivalent matrix that we need.

Suppose $R=\left(r_{i j}\right)_{m \times m}$ is the fuzzy matrix, for any $\lambda \in[0,1], \lambda$ - cut fuzzy matrix of $R$ can be obtained, that is $R_{\lambda}=\left(r_{i j}^{(\lambda)}\right)_{m \times m}$. The element of $R_{\lambda}$ is defined as

$$
r_{i j}^{(\lambda)}=\left\{\begin{array}{ll}
1, & r_{i j} \geq \lambda \\
0, & r_{i j}<\lambda
\end{array}, \quad(i, j=1,2, \cdots, m)\right.
$$

For $x_{i}, x_{j} \in X$, if $r_{i j}^{(\lambda)}=1$, object $x_{i}$ and object $x_{j}$ will be clustered into the same category at level $\lambda$. When clustering by $R_{\lambda}$ with a certain $\lambda$, classification results is the equivalent classification at level $\lambda$. 


\section{Power System Black-start Zone Partitioning Algorithm}

Black-start zone partitioning is aimed at partitioning the black-start units, units without start capacity, important substations and loads into different areas by a reasonable way. Principles should be taken into account when partitioning can be summarized as follows [13, 14]:

a) Number of voltage conversions during the blackstart process should be less than limit, so as to reduce the possible of overvoltage that is caused by unloaded lines charging.

b) Limiting the length of the path to decrease the capacitive load and then reduce the probability of generator self-excitation.

c) Limiting the number of substation involved in the black-start process to reduce the recovery operation and then reduce the risk of recovery program and the recovery time.

d) The minimum load required to start the unit without self-starting capability should be less than the maximum output of the black-start power. With this condition, the capacity of the unit that starts secondly should be chosen as large as possible.

e) Loads that have an important impact on the national economy should recover as soon as possible, hence, genrators supplying to important consumers should be restored as fast as possible.

When limiting the time-consuming of restoring the black start units, the lines and the units without black-start capacity.

\subsection{Characteristic Indexes Extracting}

The essence of black-start zone partition is to divide nodes of black-start unit, other units, important substations and important loads into different partitions by reasonable method so that each partition could carry out restoration at the same time. To adapt to algorithm proposed, the following factors are simplified when extracting the features indicators.

a) All the generators, substations and load buses in the network are abstracted as undifferentiated nodes.

b) Ignore the importance level of load, the difference in starting characteristic of generators , the differences of time operating breakers in substation and the direction of power flow in line, while, each electric transmission line and transformer are abstracted as an unweighted segment with no direction.

c) This paper just take power plant into consideration when choose the black-start unit, however, isolated island and tie line can be regarded as generator thus the algorithm proposed is also suitable for situations that system restore with power from isolated island or tie line.

In order to make sure the black-start recovery go smoothly, there must be at least one black-start power (including black-start unit, isolated island and tie line) in each partition. Black-start power supplies the energy for restarting other generators or loads through several Intermediate substations. Taking the reliability and timeliness into account, several indicators to measure the strength of the electrical contact between the target unit, load bus bars, important substations and black-start power are as follows:

a) Physical distance from the black-start power;

b) Number of voltage conversions;

c) Number of substation involved.

These three indicators are cost indexs; the target of partition is to control the three indicators as small as possible.

The electrical distance between node $i$ and node $j$ is defined as:

$$
L_{i j}=\sum_{k=1}^{l} \omega_{i j(k)} r_{i j(k)}^{\prime} \quad(i=1,2, \cdots, m, \quad j=1,2, \cdots, n)
$$

where, $r_{i j(k)}{ }^{\prime}=\frac{r_{i j(k)}-r_{\min }}{r_{\max }-r_{\min }}, r_{i j(k)}$ is the $k$-th indicators to describe the strength of the electrical contact, $r_{i j(k)}{ }^{\prime}$ is its value after normalization, $r_{\max }, r_{\min }$ are the maximum and minimum value of $r_{i j(k)}, \omega_{i}$ is the weight of $i$-th indictor, $l$ is the number of indictors and $l=3$ at present.

Taking the electrical distance between node $i$ and node $j$ as the characteristic indicator, original data matrix can be established as follows.

$$
X=\left(x_{i j}\right)_{m \times n}, x_{i j}=L_{i j},(i=1,2, \cdots, m, j=1,2, \cdots, n)
$$

where $m$ is the number of objects of classification including power plants, substations and load bus in power system, $n$ is the number of units with self-starting capability.

\subsection{Partitioning Algorithm Flow}

The steps of black-start partition with fuzzy clustering analysis method are as follows.

a) Building the original data matrix that represents the electrical distance between each node based on formula (5) and (6);

b) Normalizing every element in the original data matrix according to formula (1);

c) Forming the fuzzy similar matrix by calibrating objects to be classified with formula (2);

d) Taking the transitive closure method when clustering every object with suitable threshold $\lambda \in[0,1]$ the $\lambda$-cut fuzzy matrix of $R$ can be formed as $R_{\lambda}=\left(r_{i j}^{(\lambda)}\right)_{m \times m}$ by formula $\lambda$ thus the partition schema is determined;

Adjusting the result can obtain the final patition 
scheme by taking the practical situation of the grid into account, such as the location of black-start resources and the result of power flow check.

\section{Simulation Example}

A simulation example has been programmed to test if the fuzzy clustering analysis is rational and effective on the IEEE-39. Supposing node 32, node 36 and node 37 are the black-start units, and there must be at least one of them in each zone to ensure every zone has capability of self-starting. Thus the set to cluster includes 36 other nodes in the IEEE-39, that is

$$
X=\{1,2, \cdots, 31,33,34,35,38,39\} .
$$

Based on the electrical distance from each node to node 32 , node 36 and node 37 , the original data matrix is formed as

$$
X=\left(x_{i j}\right)_{36 \times 3}(i=1,2, \cdots, 36, j=1,2,3)
$$

Calculated with Matlab 7.6.0, we can get the normalized matrix $Y$, the fuzzy similar matrix $R$ and the transitive closure matrix $B=t(R)=R^{16}$. By any different value of threshold $\lambda \in[0,1], \lambda$ - cut fuzzy matrix of $R$ can be got. Corresponding to each $\lambda$, the equivalent classification at level $\lambda$ is shown in Table 1 .

Table 1. Partition Result Corresponding to Different Threshold.

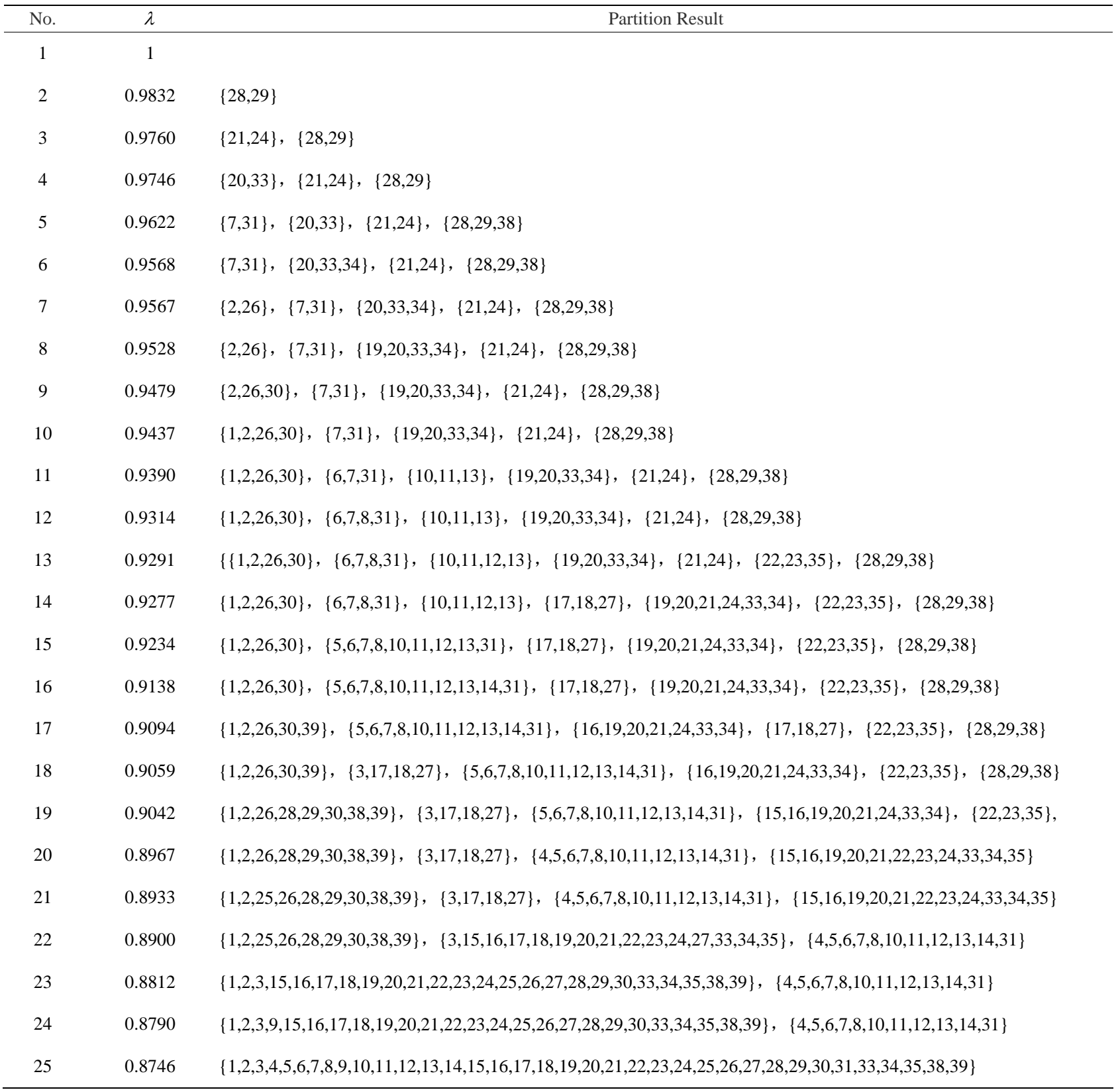


For space limitation, subsets with two nodes or more but only one node has been listed in Table 1. As is shown in Table 1, the quantity of elements with one common similarity gradually increase and the number of clustering result turn from 36 to only 1 , when threshold $\lambda$ decrease from 1 to 0.8746 . Nodes in $\{3,9,15,16\}$ join into any non-isolated class later for the electrical distance from them to the black-start power is further than to others. The reason why node 25 turned non-isolated later is that the number of long line near black-start power is too much that the node is of less similarity than others. While $\lambda=0.8900$, three zones centered by node 32 , node 36 and node 37 are formed clearly.

Partition result listed in Table $\mathbf{1}$ just means clustering result mathematicall. Powerflow verification is needed to ensure that the sum of active power should be more than sum of load in each zone by adjusting schema which failed to pass the verification. The final black-start zone partition schema is shown as is in Figure 1, where dashed lines have marked the boundaries between each zone.

As is shown in Figure 1, each of the three zones contains a black-start resource and nodes that have shorter length, less voltage conversions and less passing substations to the black-start resource than others. The result meets the principles of black-start zone partition. Adjacent rather than distant nodes are basically divided into the same zone. The characteristic indexes extracted in this paper are reasonable. During the restoration in each zone, units, with less electrical distance to the black-start resource and larger capacity on condition that the power needed while start is less than the capacity of black-start resouce, should be chosen and restart have priority. Then part of loads should be restarted gradually. In the process of recovery, system voltage and frequency stability need to be strict control, each zone should be paralleled at the right time, and all of the loads should be restarted step by step until the network is stable.

\section{Conclusions}

In this paper, a new algorithm for black-start zone partitioning based on fuzzy clustering analysis has been presented. Electrical distance between black-start resource and each of other nodes is chosen as the characteristic indicators to form the original data matrix by taking a full account of principles for black-start zone partition, and then transitive closure method has been used for clustering the nodes to get a black-start zone partitioning scheme. Simulation on the IEEE-39 has confirmed the

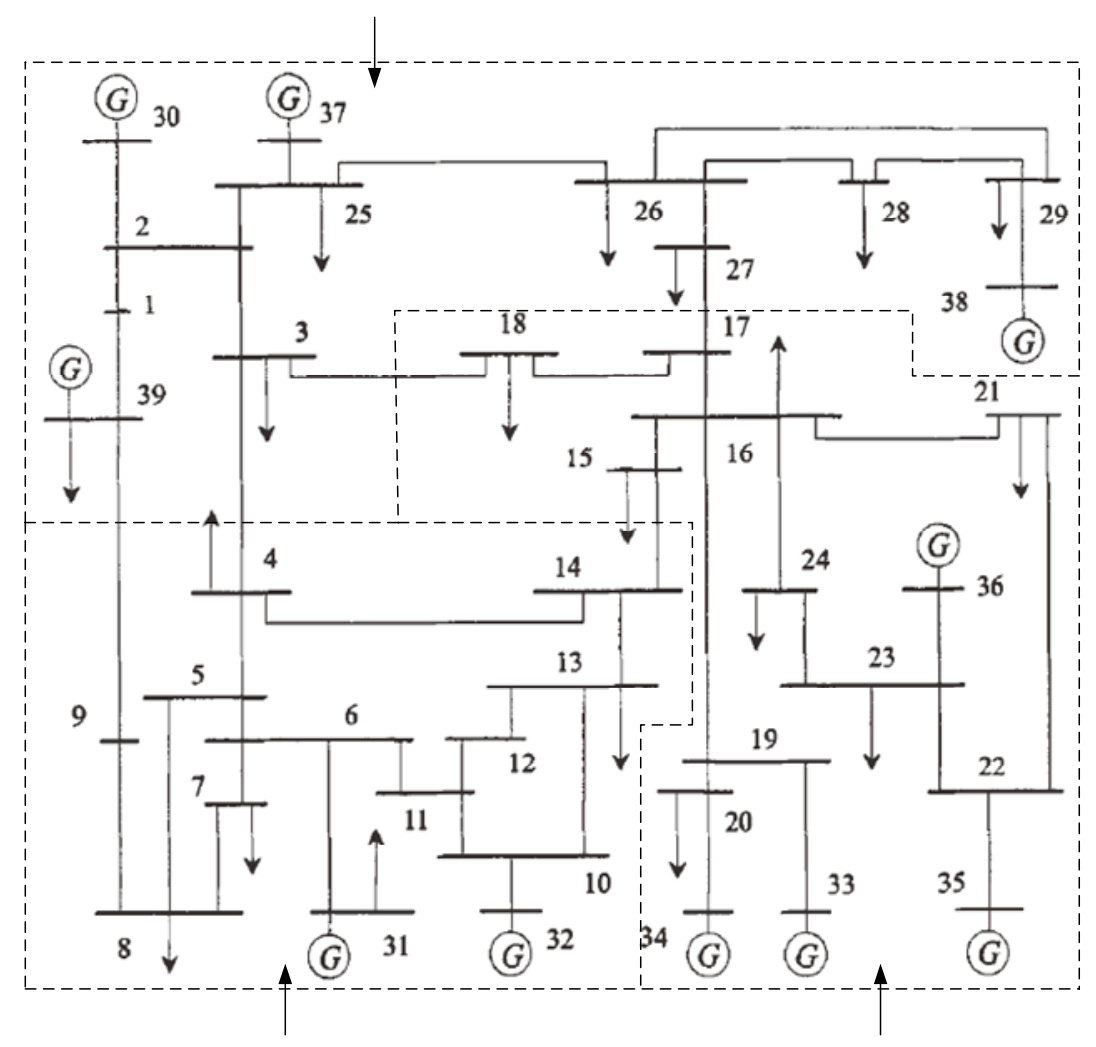

Figure 1. Black start zone partition schematic diagram. 
rationality and effectiveness of the algorithm. It is helpful to shorten the time-consuming for restarting the grid and to improve the reliability of restoration taking algorithm proposed when developing black-start zone partitioning schema after blackouts.

\section{REFERENCES}

[1] M. M. Adibi, P. Clelland, L. Fink, et al., "Power System Restoration- A Task Force Report," IEEE Transactions on Power Systems, Vol. 2, No. 2, 1987, pp. 271-277. doi:10.1109/TPWRS.1987.4335118

[2] A. Kurita and T. Sakurai, “The Power System on July 23, 1987 in Tokyo," Proceedings of the 27th Conference on Decision and Control, Vol. 3, 1988, pp. 2093-2096. doi:10.1109/CDC.1988.194703

[3] U.S.-Canada Power System Outage Task Force. Final Report on the August 14, 2003 Black out in the United States and Canada.

[4] S. Q. Tang, M. Zhang, J. S. Li, et al., "Review of Blackout in Hainan on September 26th-Causes and Recommendations,"Automation of Electric Power Systems, Vol. 30, No. 1, 2006, pp. 1-7.

[5] H. P. Liang, H. Y. Ma, X. P. Gu and Black-Start, "Network Partitioning Considering Time Limits and Subsystem Restoration Sequences," Power and Energy Engineering Conference (APPEEC), 2011, pp. 25-28.

[6] Z. Z. Lin, F. S. Wen and H. Zhou, “A New Algorithm for Restoration Subsystem Division Based on Community Structure of Complex Network Theory," Automation of Electric Power Systems, Vol. 33, No. 12, 2009, pp. 12-16.
[7] Y. S. Liu, W. C. Wu, Y. Q. Feng, et al., "Black-start Zone Partitioning Based on Ordered Binary Decision Diagram Method,” Proceedings of the CSEE, Vol. 28, No. 10, 2008, pp. 26-31.

[8] X. B. Gao, "Fuzzy Clustering Analysis and Its Applications,” Xi'an: Xidian University Press, 2004, 37-60.

[9] Z. Li, B. X. Zhou and N. Lin, "Classification of Daily Load Characteristics Curve and Forecasting of Short-term Load Based on Fuzzy Clustering and Improved BP Algorithm,” Power System Protection and Control, Vol. 40, No. 3, 2012, pp. 56-60.

[10] H. Lan, G. G. Zheng, X. L. Sun, et al., "Research of Power Transformer Fault Diagnosis Based on Vague Clustering Analysis," Electrical Measurement \& Instrumentation, Vol. 48, No. 1, 2011, pp. 46-49.

[11] R. Fu, Y. S. Qiu and Y. Li, “A Fuzzy Clustering Method for Price Partition Based on the Sensitivity of Node Price,” Power Demand Side Management , Vol. 9, No. 3, 2007, pp. 19-22.

[12] Y. Wang, J. C. Peng, Y. Q. He, et al., “Application of Fuzzy Clustering in Sencondary Voltage Control Partitioning,” RELAY, Vol. 36, No. 11, 2008, pp. 28-32.

[13] Power System Restoration Working Group, "Special Considerationsin Power System Restoration," IEEE Transon Power Systems, Vol. 7, No. 4, 1992, pp. 1419-1427.doi:10.1109/59.207363

[14] Y. W. Gao, X. P. Gu, Y. Liu, et al. “Automatic Derivation and Assessment of Power System Black-start Schemes," Automation of Electric Power Systems, Vol. 28, No. 13, 2004, pp. 50-54. 